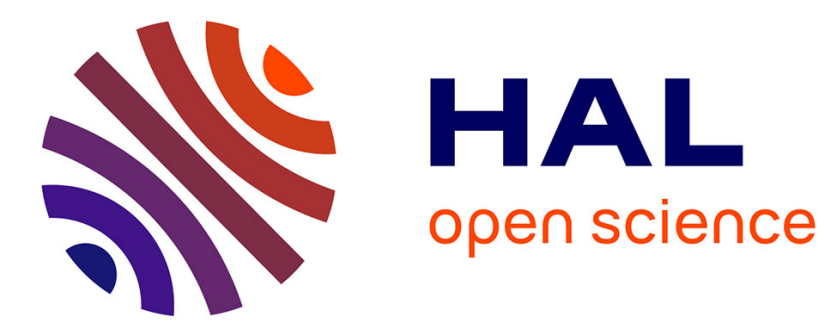

\title{
Les anciennes carrières de la ville de Morlaix et de ses abords
}

Louis Chauris

\section{To cite this version:}

Louis Chauris. Les anciennes carrières de la ville de Morlaix et de ses abords. Annales de Bretagne et des Pays de l'Ouest, 2019, 126 (4), pp.7-24. 10.4000/abpo.4753 . hal-02486200

\section{HAL Id: hal-02486200 \\ https://hal.univ-rennes2.fr/hal-02486200}

Submitted on 20 Feb 2020

HAL is a multi-disciplinary open access archive for the deposit and dissemination of scientific research documents, whether they are published or not. The documents may come from teaching and research institutions in France or abroad, or from public or private research centers.
L'archive ouverte pluridisciplinaire HAL, est destinée au dépôt et à la diffusion de documents scientifiques de niveau recherche, publiés ou non, émanant des établissements d'enseignement et de recherche français ou étrangers, des laboratoires publics ou privés. 


\title{
Les anciennes carrières de la ville de Morlaix et de ses abords
}

\author{
Louis CHAURIS \\ Directeur de recherche au CNRS, retraité
}

Grandissant au pied de son château, ceint de remparts, cerné par trois communes, Morlaix, à la limite du flot, a été longtemps un port important : vers la fin du $\mathrm{XV}^{\mathrm{e}}$ siècle, la cité se classait au troisième rang des havres bretons, après Nantes et Saint-Malo'. À l'évidence, la somptuosité de ses édifices religieux, tant églises paroissiales que bâtiments conventuels, a nécessité au cours des siècles un large emploi de matériaux de construction ${ }^{2}$. La situation de la ville au fond d'un estuaire navigable a facilité, très tôt, l'apport de granites de provenance relativement distale, entre autres de l'île de Batz et de l'île Callot et du lointain district de l'île Grande ; ultérieurement, appel a été fait aussi aux granites continentaux (Le Ponthou et Guerlesquin dans le massif de Plouaret, Huelgoat dans le centre-Finistère, Aber-lldut dans le Bas-Léon, d'autres encore...). Mais la ville et ses abords possédaient aussi des roches de nature variée qui, également très tôt, ont été recherchées. Ce sont sur ces carrières proximales, aujourd'hui bien oubliées, que l'attention est ici portée, pour la première fois, dans une vue d'ensemble.

Pour des raisons économiques, les bâtisseurs avaient tout intérêt à extraire à proximité les matériaux pondéreux dont ils avaient besoin. Dans ce but, de nombreuses carrières ont été ouvertes tant à Morlaix même que dans les communes limitrophes: Ploujean (aujourd'hui rattaché à Morlaix), Plourin, Saint-Martin-des-Champs, voire Plouigneau et Garlan. Nos informations sur ces carrières proviennent de sources diverses : données archivistiques, éparses, le plus souvent découvertes fortuitement ;

${ }^{1}$ L'histoire de Morlaix est trop connue pour que nous nous y attardions ici. Pour une vue d'ensemble, se référer à l'exposé clair et précis de TANGUY, Jean, Morlaix, Atlas historique des villes de France, Paris, CNRS, 1986. Pour plus de détails, se reporter aux ouvrages suivants: DAUMESNIL Joseph et AlLIER Adolphe, Histoire de Morlaix, Marseille, Laffitte Reprints, 1976, 553 p. ; LE JEAN, Guillaume, Histoire politique et municipale de la ville et de la communauté de Morlaix, Morlaix, 1846, 260 p. ; Le GuENNEC, Louis, Le Finistère monumental, t. 1, Morlaix et sa région, 1979, 416 p. ; DARSEL, Joachim, Histoire de Morlaix, des origines à la Révolution, Rennes, 1942, 185 p. ; LeguAY, Jean-Pierre, "Le Léon, ses villes - Morlaix au Moyen Age », Bulletin de la Société archéologique du Finistère, CVI, 1978, p. 103-160 ; LE CLECH, Marthe, Morlaix, t. I, Morlaix, 1988, 120 p. Consulter aussi le numéro des Cahiers de I'Iroise intitulé : Ce vieux Morlaix, $35^{\mathrm{e}}$ année, $\mathrm{n}^{\circ} 2$, 1988, p. 62-121.

${ }^{2}$ CHAURIS, Louis, « Cinq siècles d'histoire inscrits dans la pierre : les trois églises paroissiales du vieux Morlaix », Revue archéologique de l'Ouest, 12, 1995, p. 159-170 ; du même auteur : "Histoire des couvents de Morlaix vue à travers leurs pierres », in : Bretagne, art, négoce et société de l'Antiquité à nos jours. Mélanges offerts au professeur Jean Tanguy, 1996, 520 p., cf. p. 289-303. 
références bibliographiques soumises généralement aux mêmes aléas ; microtoponymie tant en breton ( " mengleuz » = carrière) qu'en français (" rue de la carrière ") ; cartes géologiques : feuille "Morlaix " au 1/80 000, due à Charles Barrois (1905), particulièrement intéressante ; feuille "Morlaix " au 1/50 000 (1981); carte au $1 / 25000$ de I'IGN ; photographies aériennes examinées en stéréoscopie ${ }^{3}$; en sus de ces sources, une longue pratique du terrain a permis de découvrir des sites aujourd'hui masqués. Car, à présent, toutes les exploitations sont abandonnées, plusieurs ont totalement disparu, d'autres sont plus ou moins comblées, noyées ou bâties (fig. 1).

À l'aide de ces informations, il a été tenté de réaliser ici un panorama de ces anciens sites d'extraction regroupés selon leur nature pétrographique, tant en envisageant leur importance relative et les modalités de leur emploi (pierres de taille, moellons, ardoises, matériaux de viabilité ...), qu'en présentant quelques exemples de mise en œuvre à Morlaix, dont la longue histoire va être ainsi éclairée, jusque dans ses tréfonds, d'une manière originale restée encore peu abordée.

\section{De sombres schistes omniprésents}

Morlaix est construit sur des schistes, entaillé dans ces roches qui percent partout sur les versants abrupts du Queffleuth et du Jarlot confluant du Dossen où s'est établi le port. Fréquemment, ces schistes sont caractérisés par l'alternance indéfiniment répétée de lits pélitiques bleu-noir et de lits gréseux grisâtres, d'où leur appellation de " schistes zébrés ». À ces schistes sont associées des formations tuffacées d'origine volcano-sédimentaire naguère dénommées par le comte de La Fruglaye (1766-1849) "grès des houillères ". Ces schistes renferment souvent des nodules de quartz blanchâtre extraits en même temps que l'encaissant et employés avec lui. Certains cristaux pélitiques sont constellés de cristaux d'andalousite résultant du métamorphisme de contact des intrusions granitiques (infra).

De nombreuses carrières, dont les fronts de taille subverticaux sont souvent encore bien observables, entaillent les flancs des vallées précitées. La plus célèbre est sans doute celle de Troudousten dont la toponymie conserve encore le souvenir : "rue de la carrière ». C'est ce que laisse entendre l'ingénieur chargé de la construction des écluses du port (1846-1855) ${ }^{4}$ : "La seule carrière dans laquelle on ait quelque probabilité de trouver [des moellons de choix] est celle de Troudousten ", qualifiée comme " la meilleure, la plus abondante de celles qui fournissent aux constructions de la ville ». En fait, les carrières ouvertes dans les schistes fournissaient essentiellement des " moellons ordinaires" et nettement plus rarement des "moellons de choix ». À titre d'exemple : au début de la construction des écluses du port, les carrières situées en bordure du Dossen avaient fourni $2500 \mathrm{~m}^{3}$ de moellons ordinaires et seulement 51,30 $\mathrm{m}^{3}$ de moellons de choix, soit un rapport de l'ordre de $1 / 50^{\mathrm{e}}$. Dans le même rapport, l'ingénieur indique que c'est inutilement qu'il a " fait attaquer la carrière de Pennelé, située sur le bord de la rivière de Morlaix, à 3000 mètres en aval de la ville. Sur plus de

\footnotetext{
${ }^{3}$ Photographies aériennes « Mission Ouessant-Guingamp », 1952, 0216-0816, 130-131-132.

${ }^{4}$ Arch. dép. du Finistère, $4 \mathrm{~S}$ supplément 49.
} 
$1000 \mathrm{~m}^{3}$ extraits de cette carrière, il ne se trouve pas $20 \mathrm{~m}^{3}$ de moellons de choix » (le rapport est ici à peu près le même, à savoir $1 / 50^{\mathrm{e}}$ ).

Les sites d'extraction peuvent être regroupés en différents ensembles:

- sur le versant occidental du Dossen, dans la ville même, quai de Léon, cachés derrière des maisons; au droit des écluses; à l'emplacement de l'ancienne usine à gaz ; aux environs de Pennelé... (photo 1) . Par ailleurs, dans les terres du couvent de Cuburien, très anciennes extractions du " grès des houillères ";

- sur le versant oriental du Dossen, en sus de Troudousten, à proximité des écluses, carrière occupée à présent par un immeuble, avec fronts de taille " rafraîchis " pour accroître l'emplacement; derrière la " maison Penanault» (XVII siècle); sur le versant, dans le côté droit de la route vers Lanmeur ;

- sur la rive droite du Jarlot, en bordure des allées du Poan Ben, fronts de taille des carrières dissimulées derrière des immeubles élevés sur la sole ; l'exploitation d'une carrière en ce point en 1858 est attestée par une lettre de l'entrepreneur Queinec, datée du 15 mars, demandant « la faculté de prendre des pierres de choix dans les carrières des environs de la ville dans le cas où il serait reconnu que la carrière du terrain des écoles, en bordure du Jarlot, ne pourrait pas en fournir une quantité suffisante ". Effectivement, la faible étendue de ladite carrière allait conduire l'entrepreneur à solliciter l'autorisation « de prendre quelques $\mathrm{m}^{3}$ de moellons de choix dans la carrière de Troudousten où il s'en trouve de très belles en ce moment $»^{6}$. Plus haut, dans le couvent des ursulines, il est question d'une perrière située dans l'enclos, exploitée pour la construction de la chapelle, bénie en $1659^{7}$.

Des précisions chiffrées sont parfois apportées par les archives. En voici un exemple remontant à 1856, relatif à la " construction d'un mur de soutènement du terrain des écoles le long de la rivière du Jarlot, avec une rampe pour communiquer avec la rivière ${ }^{8}$ : achat et extraction de moellons schisteux de choix des carrières des environs de la ville : prix au $\mathrm{m}^{3}$ à la carrière $(2,25 \mathrm{~F})$ et transport depuis la carrière $(1,00 \mathrm{~F})$; dans les mêmes conditions, le prix des moellons ordinaires revient respectivement $1,50 \mathrm{~F}$ et $1,00 \mathrm{~F}$. Ces prix sont à comparer au prix du $\mathrm{m}^{3}$ de la pierre de taille brute en granite de l'île Grande rendu à pied d'œuvre, à savoir 21,25 F. Un autre exemple, d'une dizaine d'années antérieur : selon le devis en date du 2 septembre 1845 concernant l'exécution des écluses, le prix du $\mathrm{m}^{3}$ de moellons de choix s'élève à $3,00 \mathrm{~F}$ $\left(2,20 \mathrm{~F}\right.$ pour l'extraction, $0,80 \mathrm{~F}$ pour le transport ; le prix $\mathrm{du}^{3} \mathrm{~m}^{3}$ de moellons ordinaires est de $1,70 \mathrm{~F}$ (respectivement 0,90 et $0,80 \mathrm{~F})^{9}$.

Un document remontant aux alentours de 1675 évoque une " montagne perrier » ( « perrier » pour " perrière » ou « carrière »), située entre le couvent des capucins et le quai de Tréguier (rive droite du Dossen), indiquant ainsi sans ambiguïté l'habitus abrupt

${ }^{5}$ Arch. dép. du Finistère, 8 S 11. En l'occurrence, il s'agit de l'autorisation (1892) d'ouvrir une carrière dans un terrain dépendant de l'usine à gaz, pour extraire du moellon. Le document localise la carrière.

${ }^{6}$ Arch. mun. de Morlaix, 1858 (archives non classées).

${ }^{7}$ Daniellou A., 1980, À travers 340 ans d'histoire. Les Ursulines de Morlaix, Morlaix, 226 p.

${ }^{8}$ Arch. municipales de Morlaix, 1856 (archives non classées).

${ }^{9}$ Arch. dép. du Finistère, 4 S suppl. 49. 
de l'excavation ouverte dans les schistes à flanc de coteau ${ }^{10}$. L'ancienneté des exploitations dans les schistes de part et d'autre de la Rivière de Morlaix est également confirmée par un décret de justice daté de $1726^{11}$. En l'occurrence, il s'agit de la curieuse histoire d'une jeune fille, violée à plusieurs reprises dans les carrières tant « du costé de Saint-Martin » qu'« au-delà des Capucins ». En 1881, il est question d'une carrière située entre le bassin à flot et la nouvelle route départementale vers Lannion ${ }^{12}$.

Les travaux publics pouvaient aussi livrer, indirectement, des pierres. Une première illustration de cette modalité, en 1771, concerne l'escarpement d'un rocher sur la terre du château de Keranroux, rive droite du Dossen, " pour y établir un quai de halage et la chaussée en y employant les pierres qui en proviendraient ${ }^{13}$ ". Un second cas, nettement plus important, s'est produit lors du percement des tranchées pour le passage de la voie ferrée Paris-Brest, achevée en 1865, de part et d'autre du viaduc, qui ont fourni un volume considérable de blocs abattus à la mine et remployés en remblais pour ladite voie ; cette modalité a encore eu lieu lors de l'établissement de la voie d'accès, de la gare au port, ouverte en 1906.

Les schistes locaux ont été si largement utilisés qu'on hésite quelque peu devant le choix des exemples. Leur habitus souvent zébré leur confère un aspect esthétique aussi original qu'indéniable. Leur emploi est prépondérant dans l'édification des hauts murs limitant les propriétés (le long de la rue Sainte-Marthe, le mur du carmel montre de grands moellons atteignant parfois plus de deux mètres de long) ou soutenant les jardins en terrasse ( "combot »). II est également omniprésent dans les demeures ellesmêmes, le granite, en pierres de taille étant alors réservé à l'entourage des ouvertures. Fréquemment, les moellons sont posés directement sur les affleurements schisteux, s'intégrant ainsi remarquablement dans l'environnement. En fait, les exemples de mise en œuvre des schistes sont de toutes les époques : déjà au XIII ${ }^{e}$ vsiècle pour les parties les plus anciennes de l'église des jacobins, avec le granitique albitique de Ploujean (infra) ; au $\mathrm{XVI}^{\mathrm{e}}$ siècle pour la maison dite de la Duchesse Anne ; à la fin du $\mathrm{XVI} \mathrm{I}^{\mathrm{e}}$ ou au début du XVII ${ }^{\mathrm{e}}$ siècle pour "Penanault » avec le granite rose de l'île Grande et plusieurs demeures de la rue longue ; aux $X \mathrm{VIII}{ }^{\mathrm{e}}$ et $\mathrm{XIX}{ }^{\mathrm{e}}$ siècles; et encore dans la seconde partie du $X X^{e}$ siècle (ancienne chambre de commerce avec le granite du Huelgoat ; chapelle Notre-Dame-des-Anges). Les nodules quartzeux, extraits en même temps que les schistes encaissants, mais impossibles à façonner, ont été employés tels quels dans les vieux murs qu'ils éclaircissent curieusement par leur blancheur.

Le " grès des houillères ॥ mérite aussi quelques remarques. II a pu être utilisé en pierre de taille dans l'église des jacobins. Toutefois, c'est surtout à Cuburien qu'il a été employé avec abondance et magnificence ${ }^{14}$. Le comte de La Fruglaye avait remarqué que cette roche se travaillait avec une grande facilité. Par lui, on savait déjà qu'une partie des pierres de la chapelle de Cuburien (première partie du XVI ${ }^{e}$ siècle) avait été

${ }^{10}$ Arch. nat., série $P$, Morlaix, $n^{\circ} 1654$.

${ }^{11}$ Arch. dép. du Finistère, $7 \mathrm{~B}$ art. 71.

${ }^{12}$ Centre généalogique du Finistère, Le Lien, n 109, mars 2009.

${ }^{13}$ Arch. mun., Morlaix, série DD (archives en cours de classement).

${ }^{14}$ ChAuRIS, Louis, "Les bâtiments du couvent de Cuburien près de Morlaix, reflets de l'utilisation pluriséculaire de la pierre en Bretagne », Bulletin de la Société archéologique du Finistère, CXXV, 1996, p. 169-197. 
façonnée dans le matériau - ce que nos observations ont pleinement confirmé - et qu'il avait été extrait dans l'enclos même du couvent, ce qui souligne dans ce cas, sa provenance toute proximale ${ }^{15}$. On l'observe aussi bien en pierres de taille dans les contreforts et en moellons dans les élévations qu'en éléments exigeant un façonnement particulier (bénitier à deux cuves, inscription en lettres gothiques portant le millésime de la fondation de la chapelle...); ici, la roche prend la place occupée très souvent en Basse-Bretagne, dans ces domaines, par le kersanton.

\section{De clairs granites albitiques}

Les schistes de Morlaix sont recoupés par deux pointements granitiques de composition fort originale, caractérisés par l'abondance de cristaux automorphes d'albite, d'où leur nuance blanchâtre; toutefois, la décomposition météorique de l'arsénopyrite (« mispickel ») qui, parfois, les constelle, entraîne l'apparition de taches brunâtres. L'un des pointements affleure à Kerscoff aux portes même de la ville (photo 2 ); il est fréquemment arénisé et alors recherché comme "sable » pour les constructions. Deux grandes carrières sont encore visibles, en bordure de la rue de Kerscoff d'une part et de la rue de la Vierge Noire d'autre part ; la première est à présent presque comblée, la seconde en grande partie bâtie. L'autre pointement, plus important constitue la butte du "Menez Plouian " à environ quatre kilomètres du centre de Morlaix. Le grand nombre de points d'extraction qui, véritablement, " truffent » le Menez s'explique à la fois par la facilité du façonnement de la roche et par la proximité de Morlaix, et ce d'autant plus que la production pouvait être facilement acheminée par voie d'eau, en remontant le Dossen jusqu'au centre de la cité.

La «nue et fauve colline » du Menez Plouian qu'évoquait au début du $X x^{e}$ siècle L. Le Guennec est aujourd'hui, en grande partie, recouverte par une végétation touffue ; c'est dire que la reconnaissance des anciennes perrières s'est avérée difficile. Nos explorations ont établi que la colline avait été occupée par de nombreux sites d'extraction présentant différents caractères typologiques :

- exploitation en surface des blocs dégagés de leur manteau arénique. Cette méthode, très répandue dans le passé, ne nécessitait pas l'enlèvement de mort-terrain diminuant ainsi considérablement les coûts - et permettait l'obtention de roches saines de belles dimensions. Ici, le travail préparatoire avait été effectué par la nature ellemême, favorisé par le relief du Menez qui avait provoqué l'entraînement des parties meubles le long des pentes ;

- dégagement des parties saines du granite encore emballées dans leur gangue d'arène. Les travaux de déblaiement étaient facilités par la texture meuble de l'encaissant ; l'arène était également utilisée, remplaçant avantageusement le sable salé des grèves ;

- extraction du granite en profondeur dans de véritables carrières. Cette modalité d'exploitation, beaucoup plus difficile et plus onéreuse, nécessitait une bonne connaissance de la structure de la roche, en particulier l'espacement entre les

\footnotetext{
${ }^{15}$ CHAURIS, Louis, « Un pionnier des Sciences de la Terre en Bretagne occidentale : le comte de La Fruglaye (1766-1849) » Bulletin de la Société archéologique du Finistère, CXXIII, 1994, p. 391-404.
} 
diaclases. Les fronts de taille encore conservés montrent la présence de grandes masses rocheuses sans défaut : tel est le cas des vieilles excavations ouvertes un peu à l'est du manoir de Coat-Moguer.

Au total, au Menez, les sites d'extraction paraissent avoir été de faible extension individuelle; les dimensions restreintes des chantiers étaient compensées par leur nombre, très certainement plusieurs dizaines ; en fait, les « rompeurs " attaquaient la roche partout où elle affleurait dans de bonnes conditions d'exploitabilité. Au cours de la dernière guerre, le Menez, qui constituait un point stratégique commandant la baie de Morlaix, a été fortifié par les Allemands qui ont tiré parti des excavations pour y implanter leurs blockhaus... Originale destinée de carrières abandonnées.

C'est à ces granites albitiques - et surtout à celui du Menez - que se rapportent les pierres de taille des plus anciens monuments de la cité et de ses abords, sans qu'il soit toujours possible de les rattacher à l'un ou l'autre pointement. Parmi d'autres, citons ici les piliers ( $\mathrm{Xl}^{\mathrm{e}}$ siècle) de l'église de Ploujean; les éléments les plus anciens (XIII ${ }^{e}$ siècle) de l'église des jacobins ; les piliers octogonaux (XIV siècle) de l'église de Ploujean ; les restes des remparts de Morlaix ( $x v^{e}$ siècle); le porche méridional (fin du $\mathrm{XV}^{\mathrm{e}}$ siècle) de l'église Sainte-Melaine à Morlaix ; l'ossuaire de Ploujean et la chapelle Sainte-Geneviève au $\mathrm{XVl}^{\mathrm{e}}$ siècle ; les portes jumelées de la " fontaine des Carmelites » au $X V I^{\mathrm{e}}$ siècle... ${ }^{16}$. Ultérieurement, leur utilisation se raréfie et, le plus souvent, il s'agit alors de remploi. Ces exemples qu'il eût été facile de multiplier suffisent pour établir, sans ambiguïté, que les perrières ouvertes dans le granite albitique ont été actives pendant plus d'un demi-millénaire, cas d'une longévité assez remarquable pour être soulignée.

\section{Microgranites albitiques}

Les pointements de Kerscoff et du Menez ont émis, lors de leur intrusion, de nombreux filons satellites de même composition, mais de texture différente, à savoir des microgranites albitiques, généralement de faible puissance, parfois d'ordre métrique seulement, dans les schistes encaissants qu'ils recoupent "à l'emporte-pièce ". D'excellents affleurements sont visibles à Morlaix même, sur les deux rives du Dossen (photo 3). Ces microgranites, qui présentent parfois des cernes subconcentriques brunâtres d'hydroxyde de fer, sont utilisés en moellons en association avec les schistes, comme le montrent de nombreuses constructions dans le secteur nord-est de Morlaix où les filons sont particulièrement fréquents; comme les schistes, leur emploi est ici tout proximal.

\section{Microgranite porphyrique}

Aux environs de Ploujean, les schistes de Morlaix sont recoupés par des filons de microgranite, de quelques mètres de puissance, caractérisés par la forte dimension des feldspaths potassiques (jusqu'à $8 \mathrm{~cm}$ ) et, dans une moindre mesure, de quartz bi-

${ }^{16}$ ChAURIS, Louis, « Une belle pierre oubliée : le granite albitique de Ploujean près de Morlaix (Finistère) », Revue archéologique de l'Ouest, 10, 1993, p. 141-155 
pyramidés subcentimétriques, de nuance mauve, dans un fond grisâtre à rougebrunâtre. Cette roche a été exploitée pour les constructions locales dans de petites carrières, voire débitée aux dépens de grosses boules isolées à la surface du sol, qui livraient des moellons, mais aussi, dans quelques cas, des pierres de taille. En fait, elle doit son ancienne célébrité au comte de La Fruglaye qui l'avait découverte dans le parc de son domaine à Keranroux en Ploujean et qui, après un polissage, excellent, l'avait employée pour pendule et cheminée, à la manière du marbre. Charles Barrois croit savoir que c'est en l'honneur de ce savant que la roche de Keranroux avait été dénommée fruglite ${ }^{17}$.

\section{Schistes et quartzites dévoniens}

Au sud du bassin carbonifère de Morlaix, à dominante schisteuse, affleure une vaste formation schisto-quartziteuse datée du dévonien inférieur. Les roches ne pouvant livrer aux constructions rurales voisines que des moellons médiocres, ont surtout été exploitées pour l'empierrement. De nombreuses carrières s'échelonnent d'ouest en est depuis Kerolzec en Saint-Martin-des-Champs jusqu'au Glaira en Plouigneau, en passant par le Merdy, Castoret, Parc-au-Duc, Berlingar. Seules quelques-unes seront succinctement présentées :

- Kerolzec était, de loin, la plus importante carrière des abords morlaisiens. Son intérêt minéralogique repose sur la présence de veinules quartzeuses minéralisées. La pyrite, très abondante, est massive, grenue, en plages centimétriques souvent fracturées ; elle est parfois altérée en goethite. La marcassite, fréquente, est associée à la pyrite ; elle peut montrer des structures radiées. La chalcopyrite, rare, s'altère en covellite. L'arsénopyrite, assez fréquente, renferme des traces de pyrrhotite ${ }^{18}$. L'immense excavation est partiellement transformée en décharge contrôlée ; mais une vaste zone, aujourd'hui ennoyée, forme une étendue d'eau aux rives abruptes, évoquant un lac de montagne dont on peut regretter qu'il ne soit pas aménagé (photo 4).

- Le Glaira, en Plouigneau, mais seulement à environ $3 \mathrm{~km}$ à l'est-sud-est de Morlaix, un peu au sud de la voie ferrée Paris-Brest, était aussi une importante exploitation. Pour le minéralogiste, elle offre l'attrait de la présence, dans les schistes, de plusieurs minéraux de métamorphisme : andalousite en longs cristaux bien formés ; grenat ; sillimanite fibreuse et staurotide en petits individus automorphes (photo 5).

- Le Merdy, à la sortie sud de Morlaix, en bordure du Queffleuth. La carrière exploitait des quartzites à grain très fin, gris sombre, en gros blancs, recoupés par d'innombrables filonnets de quartz blanc à éclat gras. Les fronts de taille sont encore bien dégagés ; la sole de la carrière est aménagée en jardin... En face du Merdy, à Roc'h Bleiz, il était cru naguère que le quartz était aurifère ; selon toute probabilité, la pyrite - sulfure de fer jaune brillant - y avait été prise pour de l'or ${ }^{19}$. C'est dans la carrière du Merdy que le docteur Le Hir, pionnier - avec le comte de La Fruglaye - de la

17 BARRoIs, Charles, "Compte rendu de l'excursion du 28 août aux environs de Morlaix », Bulletin de la Société géologique de France, $3^{\text {e }}$ série, T. XIV, 1886, p. 244-254.

${ }_{18}$ Pyrite = sulfure de fer $;$ marcasite = sulfure de fer $;$ chalcopyrite $=$ sulfure de fer et de cuivre $;$ covellite $=$ sulfure de cuivre $;$ goethite $=$ hydroxyde de fer $;$ arsénopyrite (anciennement « mispickel ») = arséno-sulfure de fer ; pyrrhotite = sulfure de fer. 
géologie dans la région morlaisienne, avait découvert des fossiles, étudiés ultérieurement par Charles Barrois en $1886^{20}$ et Charles Delattre en $1952^{21}$.

Au sud de la guirlande précitée, des exploitations étaient ouvertes à Bohast et à Penhoat dans les formations schisto-quartziteuses affectées par le métamorphisme de contact lié à de petites intrusions granitiques.

Deux autres carrières doivent encore être signalées ici. La première, entièrement comblée, était située à Morlaix même, au lieu-dit Parc-au-Duc. Elle livrait des schistes criblés de petits cristaux d'andalousite - liés au métamorphisme de contact d'une intrusion granitique ici encore en profondeur - utilisés en moellons, en particulier pour les constructions du voisinage. L'autre, en grande partie envahie par la végétation, était ouverte à Toulgoat dans la partie occidentale de l'immense commune de Plouigneau, à moins de quatre kilomètres du centre de Morlaix ; elle exploitait un grès - dont l'âge est encore discuté - pouvant fournir des moellons et des matériaux de viabilité.

\section{La syénite quartzifère de Pont-Pol}

Un peu au sud de Morlaix, les formations dévoniennes sont recoupées par plusieurs pointements d'une roche exceptionnelle - à savoir une syénite quartzifère caractérisée par son fort pourcentage en feldspath potassique ( 55-60\%), sa faible teneur en quartz $(\sim 10 \%)$ et une riche dissémination d'allanite en petits cristaux $(\sim 2 \mathrm{~mm})$, induisant une forte radioactivité due à une teneur élevée en thorium ${ }^{22}$. La roche offre un grain fin (1-3 $\mathrm{mm}$ ) et une teinte légèrement mésocrate.

Plusieurs pointements ont été reconnus: Pont-Pol, Luzuria, Moulin-Vieux, Kerloaguen, Penrugaro, tous de faible dimension ; le plus important, celui de Pont-Pol, dépasse légèrement $2 \mathrm{~km}$ sur $0,7 \mathrm{~km}$. L'arénisation est souvent intense, atteignant plus de 10 mètres d'épaisseur à Penrugaro ; les zones saines se limitent alors à des boules isolées. Ces syénites quartzifères ont été exploitées dans le passé ainsi que l'attestent aujourd'hui de nombreuses constructions; cette roche joue un rôle majeur dans la façade principale (fin du $X V I^{\mid}{ }^{e}$ siècle) de l'église Saint-Martin, en éléments de grand appareil ; c'est encore elle qui a été recherchée, pro parte, pour la tour (1851-1855) ; les pierres étaient alors extraites de la carrière de Keryvoas près de Pont-Pol, mise à la disposition de la fabrique par $M$. de Lescoët, du château de Lesquiffiou ${ }^{23}$. À quelque distance du château, la «ferme modèle » (1864) est un ensemble remarquable avec ses piliers monolithes d'environ trois mètres de haut et ses colossales mangeoires ; la

${ }^{19}$ LA Herblinais, A. J. D., de, Promenades à travers le pays de Morlaix, 1908, 88 p., réimpr. édit. Kornog, Morlaix, 1986.

${ }^{20}$ BARROIS, Charles, "Compte rendu de l'excursion... », loc. cit.

${ }^{21}$ Delattre, Charles, "Recherches sur le Dévonien et le Carbonifère de la région de Morlaix ». Mémoires du Service de la Carte géologique de la France, 1952, 125 p.

${ }^{22}$ CHAURIS, Louis, "Syénites quartzifères riches en thorium et terres rares en Bretagne septentrionale », Géologie de la France, 1992, 2, p. 17-22 ; Idem, "Une pierre oubliée: la syénite quartzifère de Pont-Pol près de Morlaix en Bretagne (France) », Bulletin Musée de la Pierre, Maffle, Belgique, 10, 1995, p. 29-51.

${ }^{23}$ Arch. dép. du Finistère, Église Saint-Martin, Morlaix, V, dépôt 22. 
tradition a conservé le souvenir du jour où l'on avait chargé à la carrière de Goaz-Vizien un énorme rocher acheminé à pied d'œuvre par un attelage de 30 chevaux ! La syénite a été également largement employée pour la mairie de Morlaix (1838-1849) et dans plusieurs immeubles de la ville.

Les carrières de Pont-Pol avaient reçu, en 1886, la visite, sous la direction de Charles Barrois, des participants à l'excursion de la Société géologique de France ${ }^{24}$. "La Société reconnaît [...] que les blocs exploités sont isolés sous forme de boules, parfois énormes, dans une arène [...] résultant de la décomposition sur place, opérée suivant les joints » de la roche. Dans le pointement de Pont-Pol, la petite dimension des carrières était compensée par leur nombre.

\section{Schistes tégulaires}

Les anciennes ardoisières des environs de Morlaix, depuis longtemps abandonnées, sont encore, dans plusieurs cas, reconnaissables dans le paysage, tant par les vestiges des excavations que par les déblais de taille. Ces traces, plus ou moins bien conservées, nous renseignent à la fois sur l'importance relative des exploitations et sur les qualités (fissilité, dimension...) du matériau. Les gisements étaient situés à SaintMartin-des-Champs (Trohoat, Ty Guen, Le Penquer), à Ploujean (près de Kerantour), à Garlan (Coat ar Roc'h, Poulran...). En contrebas de Kerantour, à proximité de la profonde ria du Dourduff, la carrière, envahie par la végétation, paraît avoir présenté une certaine ampleur à en juger par le front de taille ; l'escarpement naturel primitif a contribué à faciliter l'extraction. À Coat ar Roc'h, l'exploitation s'est poursuivie entre les deux guerres en carrière souterraine ; à présent, les déblais recouvrent en partie les anciens travaux. En l'absence d'archives, il est aujourd'hui impossible de préciser d'où provenaient les ardoises employées pour les anciennes toitures à Morlaix.

Par contre, les archives renseignent parfois sur le nombre des ouvriers travaillant dans les ardoisières ${ }^{25}$. Ainsi, en 1838, le sieur R. Dumoulin, "perrayeur », fait part au maire de Garlan de son intention d'exploiter l'ardoisière de Poulran, avec un nommé Lazennec: : En tout, ils seront au nombre de cinq. Trois d'entre eux seront employés au transport des pierres hors de la carrière [ce qui suggère l'importance des déblais], un pour les extraire, un autre pour les tailler ». Les travaux sont prévus à ciel ouvert. La même année, G. Berthou, cultivateur à Garlan, se propose de continuer l'exploitation d'une ardoisière en activité dans un champ dit Parc Huella. L'exploitation comprendra « quatre ouvriers dont deux seront employés à extraire les pierres, un à les fendre et le quatrième à enlever les matières et à retirer les eaux ". Ces informations confirment qu'il s'agit de petites exploitations artisanales.

L'histoire des ardoisières de Garlan semble avoir été assez mouvementée, avec des démêlés entre les exploitants et l'Administration. Dans une lettre au préfet, le 5 octobre 1847, l'ingénieur des Mines demande "de faire cesser les dangers que présentent pour la sûreté publique plusieurs ardoisières" ouvertes dans cette commune. Aussitôt, un arrêté préfectoral (9 octobre) enjoignait aux sieurs Dumoulin et

\footnotetext{
${ }^{24}$ BARRoIs, Charles, "Compte rendu de l'excursion... », loc. cit.

${ }^{25}$ Arch. dép. du Finistère, 8 S 13 et 8 S 16.
} 
Lazennec de remblayer l'ancienne carrière abandonnée de Poulran et de garantir l'approche de la nouvelle carrière qu'ils exploitaient. Quelques années plus tard, le 6 juillet 1855, l'ingénieur en chef des Mines écrit au préfet au sujet de G. Berthou, maître carrier demeurant à Garlan, qui avait ouvert une carrière d'ardoises sans avoir produit de déclaration préalable. L'ingénieur ajoute que les travaux ne sont pas à distance légale du chemin conduisant du village de Poulran au bourg de Garlan ; il note que les terres placées en recouvrement de la roche exploitée ne sont pas coupées par un talus suffisant pour prévenir les risques d'éboulement. En conséquence, il demande d'enjoindre au sieur Berthou de cesser immédiatement toute exploitation et de combler dans le délai d'un mois la carrière qu'il a ouverte. Une quinzaine d'années plus tard, un rapport de l'ingénieur des Mines (13 juillet 1870) apporte des informations sur les travaux souterrains entrepris par le fils Berthou. L'excavation est distante de 6 mètres à partir de son orifice, du chemin conduisant de Poulran au bourg de Garlan. En continuant dans cette direction, elle arrivera « à traverser le chemin dont [elle] n'est séparée actuellement que par une distance de deux mètres environ ». Or " le massif de roche situé au-dessus de l'excavation est complètement décomposé et ne présente aucune solidité $[\ldots]$; il est donc à craindre que le sieur Berthou ne provoque un éboulement et ne compromette la sécurité de ses ouvriers ainsi que la solidité du chemin $^{26}$ ॥.

\section{Grès de Toulgoat}

Au lieu-dit La Croix-Rouge, à la limite méridionale de la commune de Garlan, un peu à l'est de Morlaix, affleure une étroite bande de grès blanchâtre dont le passage se marque encore dans le paysage par le développement des taillis. Cette formation est connue des géologues sous le nom de "Grès de Toulgoat » dont l'âge reste incertain. Ces grès étaient exploités dans de nombreuses carrières qui livraient non seulement des moellons à l'habitat rural - d'où les murs très clairs des vieilles fermes - mais aussi de bons matériaux d'empierrement.

Une lettre adressée aux autorités préfectorales en mars 1857 par le fermier travaillant les terres près de la Croix-Rouge décrit l'état des lieux, à la suite des extractions en cours : " Dans les garennes [...] de la Croix-Rouge, il est extrait [...] des pierres pour l'empierrement de la route impériale [ultérieurement R.N. 12, aujourd'hui RD 712]. L'entrepreneur de cette route exerce des ravages [...] : non seulement 3 ou 4 hectares sont le théâtre de 80 à 100 trous de carrières ॥, mais ces trous sont abandonnés si de nouvelles recherches, à proximité, paraissent plus profitables. Par ailleurs, " des parcelles de landes sont à chaque instant coupées pour y établir des emplacements de concassage, lesquels concassages détruisent de fond en comble le [...] sol et à tout jamais la végétation ». Les trous « abandonnés ne sont pas le moindrement comblés ${ }^{27}$ ».

\footnotetext{
${ }^{26}$ Arch. dép. du Finistère, 8 S 10.

${ }^{27}$ CHAURIS, Louis, "Problèmes d'empierrement des routes dans le Finistère au XIX ${ }^{\mathrm{e}}$ siècle ", Bulletin de la Société archéologique du Finistère, CXXX, 2001, p. 325-335.
} 
Dans sa réponse, l'ingénieur des Ponts et Chaussées tempère les dires du fermier, estimant qu'ils sont « pour la plupart exagérés ॥. II n'en est pas moins vrai qu'un sérieux contentieux s'était élevé entre le fermier, l'entrepreneur et les Ponts et Chaussées, soulignant que l'exploitation des carrières n'était pas sans créer des problèmes d'environnement. Aujourd'hui encore, le sol où apparaissent çà et là des blocs de grès blanc, porte essentiellement une végétation de châtaigniers, bouleaux, chênes, noisetiers, houx... II est un peu partout bouleversé par de petites excavations peu profondes, de contours irréguliers, disséminées de manière aléatoire.

Bien que succinctes, les annotations sur les anciennes carrières des environs de Morlaix permettent de formuler plusieurs remarques de portée plus générale. Quatre points semblent pouvoir être retenus :

1 - Le très grand nombre de sites d'extraction, plusieurs dizaines, et il est évident que leur nombre a dû être encore plus élevé, certains sites ayant, selon toute probabilité, échappé à nos investigations. Si l'on met à part les grandes carrières de Kerolzec et du Glaira, exploitées tardivement de façon industrielle, toutes les perrières étaient ici de faible dimension et uniquement de type artisanal. Depuis leur abandon, elles ont glissé dans le domaine silencieux de l'archéologie que ces quelques pages ont tenté de dévoiler (fig. 1).

2 - La diversité des roches exploitées, reflet de la complexité géologique du sous-sol des environs de Morlaix :

- roches sédimentaires - souvent affectées par le métamorphisme de contact des pointements granitiques : quartzite (Le Merdy ...), grès (Toulgoat), schiste ardoisier, et formations volcano-sédimentaire (grès des houillères); les schistes "zébrés " de Morlaix méritent ici une mention particulière du fait de leur originalité et de leur beauté ;

- granites et roches apparentées, remarquables par leur singularité dans l'immense panoplie bretonne : granitique albitique et syénite quarzifère (fig. 2) ;

- microgranites sodiques (albitiques) en filons rayonnant autour des pointements de Kerscoff et du Menez, et microgranite potassique de Ploujean - ou " fruglite ».

La plupart de ces roches ont été exploitées pour elles-mêmes. Toutefois, les nodules quartzeux et plus encore les minces filons de microgranite albitique mis à jour dans les carrières en même temps que les schistes encaissants, fournissaient ipso facto des matériaux d'appoint, non négligeables dans le cas des microgranites.

3 - Leur impact sur les constructions, émanations du sous-sol local. Toutefois, très tôt, appel a été fait aussi à des granites distaux. Cette double provenance, dans chaque cas multiforme, a induit dans la cité un polylithisme tant originel qu'acquis, auquel elle doit une grande part de son charme indéniable.

4 - La question des travaux de restauration. L'intérêt patrimonial de nombreuses constructions à Morlaix ne fait aucun doute. Mais encore faut-il savoir les entretenir. Lors des restaurations, il serait judicieux de faire appel aux pierres d'origine, surtout 
quand elles offrent une exceptionnelle originalité (granite albitique, schistes zébrés). Sur le Menez, il n'y aurait sans doute pas trop de difficulté à rouvrir une carrière artisanale. Les schistes zébrés posent plus de problèmes. Des architectes locaux nous ont confié les difficultés rencontrées pour obtenir cette roche, obligés qu'ils étaient de faire appel à des édifices ruinés, faute de pouvoir disposer d'une carrière ; la recherche d'un tel site apparaît être de première urgence. Une protection de l'environnement bien conçue ne doit pas se limiter au milieu naturel, mais doit aussi embrasser le cadre façonné par l'Homme au cours des siècles.

\section{LÉGENDE DES FIGURES}

Carte 1. Localisation des anciennes carrières aux environs de Morlaix.

1- Schistes (zébrés) - Moellons . 2- Quartzites et schistes - Matériaux de viabilité et moellons. 3- Schistes fissiles - Ardoises. 4- Granite albitique - Pierre de taille. 5Syénite quartzifère - Pierre de taille. 6- Grès - 7- Voie ferrée - Tranchées. Les microgranites albitiques, autour de Kerscoff et du Menez n'ont pas été figurés. Gisements parfois indiqués à titre collectif.

\section{Carte 2. Constructions ayant utilisé la syénite quartzifère de Pont-Pol.}

1- Eglise, chapelle. 2- Château. 3- Autre habitat. 4- Édifice public, ouvrage d'art. 5Divers (pilier, mur, calvaire, usine, (cf. note 22).

\section{LÉGENDE DES PHOTOGRAPHIES (Clichés Louis Chauris)}

Fig. 1 Derrières des immeubles, quai de Léon, front de taille subvertical dans les schistes.

Fig. 2 Carrière de Kerscoff. Granite albitique fortement diaclasé.

Fig. 3 Sur la rive droite du Dossen à proximité des écluses. Ancienne carrière en cours d'élargissement avant la construction d'un immeuble. Filon de microgranite albitique recoupant les schistes.

Fig. 4 Carrière partiellement ennoyée de Kerolzec.

Fig. 5 Carrière du Glaira. 